\title{
Editorial
}

\section{Sobre la prevención de enfermedades infecciosas en viajeros}

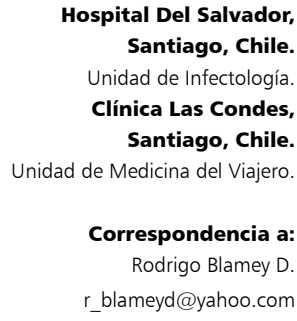

Hospital Del Salvador Santiago, Chile. Unidad de Infectología. Clínica Las Condes, Santiago, Chile. Unidad de Medicina del Viajero.

Correspondencia a: Rodrigo Blamey D. r_blameyd@yahoo.com

Es evidente que el desplazamiento de personas entre los distintos países es un fenómeno masivo y que los motivos para tales viajes son múltiples, como una expresión más de la globalización. Es así como miles de personas viajan no sólo por vacaciones, sino que también por negocios, misiones especiales u otros. Para atender las necesidades propias de este grupo de personas es que durante las últimas dos décadas se ha desarrollado la especialidad de Medicina del Viajero, cuyos conceptos son habitualmente poco conocidos para muchos médicos.

A modo de ilustrar la magnitud de pacientes potenciales en nuestro país, el año 2009 se registraron 2.895 .412 salidas de chilenos al extranjero, incluyendo las vías aérea, terrestre y marítima. Los destinos más frecuentes fueron los países de la región en Sudamérica (86\%), luego América del Norte (7,3\%), Europa $(3,7 \%)$ y el resto del mundo. Dentro de América, el destino más frecuente fue Argentina (41,9\%), luego siguieron Perú, Estados Unidos de América y Brasil. Los meses de mayor frecuencia de viajes a Sudamérica fueron febrero y enero, mientras que a Europa ocurrieron de preferencia en septiembre ${ }^{1}$.

En el último tiempo, los viajeros chilenos consultan cada vez con mayor frecuencia previo a la realización de sus viajes, y muchos no lo hacen con la debida antelación recomendada de 6 a 8 semanas, sino que en el último minuto. Aun así, debemos estar preparados para enfrentar este tipo de pacientes y ofrecerles una atención adecuada.

De esta forma, presentamos a continuación y en el número de abril, los artículos de destacados colegas infectólogos, en relación a los riesgos asociados a viajes hacia tres grandes zonas geográficas mundiales: África, Asia y Sudamérica, y finalmente un artículo en relación a viajeros especiales.

A manera de preámbulo, es necesario señalar algunos conceptos generales en relación a la Medicina del Viajero.

La evaluación médica previa a los viajes debe ser realizada por un especialista que maneje los detalles de una evaluación basada en el riesgo personal en relación al itinerario de viaje y los objetivos son la preparación eficiente del viajero mediante consejería, vacunaciones y medicamentos que ayuden a minimizar el riesgo de enfermedades o accidentes durante el viaje.

Para lograr este objetivo se debe realizar, en primer lugar, una evaluación del riesgo, de acuerdo a los antecedentes mórbidos personales y el itinerario especifico (incluyendo lugar de alojamiento y actividades a realizar); en segundo lugar, se debe comunicar en forma explícita y clara los riesgos al paciente, para lo cual es útil entregar información escrita, de tal forma que el paciente comprenda a cabalidad las indicaciones. Por último, se debe manejar los riesgos mediante la selección y administración de vacunas, medicamentos profilácticos, educación general acerca de enfermedades transmitidas por insectos y por alimentos, y otros riesgos para la salud asociados a actividades específicas, como por ejemplo la altura, deporte aventura, deportes acuáticos, exposición a infecciones de transmisión sexual (ITS), etc. Es útil además indicar un botiquín básico de medicamentos para el alivio de síntomas leves de enfermedades comunes como infecciones respiratorias altas o alergias leves. Por último, siempre discutir acerca de las alternativas de acceso a atención médica en el lugar a visitar ${ }^{2}$.

Cabe señalar, que en estudios de viajeros norteamericanos, la principal causa de muerte lo constituyeron la enfermedad cardiovascular, seguida de los accidentes; por lo tanto, estos tópicos también deben ser parte de la evaluación y consejería de los pacientes ${ }^{3}$.

Para manejar el riesgo asociado a cada viaje, sin duda, debemos estar informados en forma actualizada. Los artículos presentados no pretenden una revisión exhaustiva sino una guía para las recomendaciones de viajeros, y los médicos deberán consultar las fuentes de información adecuadas para mantenerse al día en relación a enfermedades emergentes, brotes y otras situaciones de amenaza para la salud de los pacientes como fenómenos de violencia social. Algunos sitios de internet son útiles para lograr este objetivo y contienen más información relevante para este tema. A continuación se citan algunos de ellos: 
- Salud del viajero, del Centro para el Control y Prevención de Enfermedades (CDC), de Estados Unidos: http:// wwwnc.cdc.gov/travel/destinations/list.aspx

- Noticias de brotes y amenazas en medicina del viajero (CDC) http://wwwnc.cdc.gov/travel/notices.aspx

- Organización Mundial de la Salud, página web para medicina del viajero http://www.who.int/ith/en/

- Comite Asesor en Practicas de Inmunizaciones del CDC (ACIP): http://www.cdc.gov/vaccines/pubs/ACIP-list.htm

- Epidemiology and Prevention of Vaccine-Preventable Diseases, The Pink Book, 11th Edition, May 2009: http:// www.cdc.gov/vaccines/pubs/pinkbook/default.htm

- Programa Conjunto de las Naciones Unidas para el VIH/SIDA (UNAIDS), Restricciones de viajes para pacientes con VIH: http://www.unaids.org/en/strategygoalsby2015/hiv-relatedtravelrestrictions/

- Página del gobierno de Estados Unidos acerca de la seguridad de viajes a cada país: http://travel.state.gov/ travel/travel_1744.html

- Casos del Instituto Gorgas http://gorgas.dom.uab.edu/2010cases/100809.html

Aunque conocer el riesgo real de un viajero a una determinada área es difícil, dado la variación temporal de la epidemiología de las enfermedades, la escasez de estudios bien diseñados, etc., hay alguna información acerca de las enfermedades de mayor riesgo y, en general, exceptuando la malaria, la mayoría se asocia a bajo riesgo (Tabla 1$)^{4}$.

Por otra parte, en un estudio realizado por GeoSentinel (Red de estudio colaborativo entre la Sociedad Internacional de Medicina del Viajero y el CDC) se demostró que, de acuerdo a los destinos realizados, los mayores riesgos corresponden a enfermedades febriles, diarrea, afecciones dermatológicas e infecciones respiratorias. Entre las enfermedades específicas, la malaria correspondió a la primera causa global de fiebre en los viajeros, el dengue también fue muy frecuente y en África sub-sahariana fueron relevantes las rickettsiosis ${ }^{5}$.

Por último, es preciso señalar que existen diversas guías internacionales para medicina del viajero y no siempre son concordantes, ya que se realizan en base a estudios y experiencias locales o, en algunos casos, no existe evidencia cientifica para realizar una recomendación concreta. Además, la disponibilidad de vacunas $y$ medicamentos no es universal y estos difieren entre distintos países, y nosotros no estamos ajenos a ello, por lo tanto debemos estar bien informados de las vacunas $y$ medicamentos disponibles en nuestro país al momento de hacer las recomendaciones a nuestros pacientes.

En esta dinámica área de la infectología, podemos decir que mientras más viajeros bien informados reciban una buena evaluación y consejería médica previa a sus viajes, y mientras más médicos consideren los antecedentes de los viajes al evaluar a viajeros enfermos, los viajes internacionales serán una experiencia cada vez más saludable.
Tabla 1. Estimación porcentual del riesgo de adquirir enfermedades

\begin{tabular}{llc|}
\hline Infecciosas en los viajeros & Comentario & Riesgo \% \\
\hline Diarrea del viajero & $\begin{array}{l}\text { (Escherichia coli enterotoxigénica > 15\% } \\
\text { del total) }\end{array}$ & 100 \\
\hline $\begin{array}{l}\text { Malaria } \\
\text { Influenza A o B }\end{array}$ & Sin Q.-profilaxis África Oeste & 10 \\
\hline $\begin{array}{l}\text { Dengue sintomático } \\
\text { Mordedura animal con riesgo de rabia }\end{array}$ & & 1 \\
\hline Malaria & & \\
\hline $\begin{array}{l}\text { Hepatitis A } \\
\text { Fiebre tifoidea }\end{array}$ & Con o sin Q.-profilaxis en África tropical \\
\hline Encefalitis por garrapata & Sudeste asiático, Norte, Oeste y centro & \\
\hline $\begin{array}{l}\text { Hepatitis B } \\
\text { Fiebre tifoidea (otras áreas) } \\
\text { Infección por VIH }\end{array}$ & de África & \\
\hline Accidente fatal & Austria rural & 0,01 \\
\hline $\begin{array}{l}\text { Cólera } \\
\text { Legionelosis }\end{array}$ & & \\
\hline Encefalitis japonesa & & \\
\hline $\begin{array}{l}\text { Enfermedad meningocóccica } \\
\text { Poliomielitis }\end{array}$ & & 0,001 \\
\hline Adaptada de referencia 4. & & 0,0001 \\
\hline
\end{tabular}

\section{Referencias}

1.- Turismo, Informe Anual 2009. INE-SERNATUR, Gobierno de Chile.

2.- Yellow Book, acceso online 09 enero 2011 en página web http://wwwnc.cdc.gov/travel/ content/yellowbook/home-2010.aspx
3.- Powell B, Ford C. Risk of travel, benefits of a specialist consult. Clev Clin J Med 2010; 77 (4): 246-54.

4.- Steffen R, Amitirigala I, Mutsch M. Health risk among travelers-Need for regular updates. J Travel Med 2008; 15 (3): 145-6. Acceso libre en http://onlinelibrary.wiley.com/doi/10.1111/ j. 1708-8305.2008.00198.x/full

5.- Freedman D O, Weld L H, Kozarsky P E, Fisk T, Robins R, von Sonnenburg F, et al. Spectrum of disease and relation to place of exposure among ill returned travelers. N Eng J Med 2006; 354: 119-30. 